\title{
Un enfoque multilingüe y contrastivo de la traducción fraseológica: el corpus paralelo GRAFE
}

\section{A Multilingual and Contrastive Approach to Phraseological Translation: The Parallel Corpus GRAFE}

\section{Ramón Martí SOLANo}

Universidad de Limoges

ramon.marti-solano@unilim.fr

Resumen: Este artículo presenta los resultados del análisis traductológico de una selección de unidades fraseológicas (UF) extraídas de una muestra de la obra de David Lodge y de sus traducciones en español y en francés. Se propone una tipología de los principales problemas de fraseotraducción a los que se aplica, según los casos, una metodología basada en el análisis traductológico contrastivo gracias al uso del corpus GRAFE y al programa de concordancias Paraconc. Se trata de aspectos lingüísticos que aparecen en las traducciones en un idioma meta y que pueden estar ausentes en las del otro o incluso en ambos. Hay que destacar dos fenómenos principales: por un lado, la neutralización de la información pragmática (registro de lengua, eufemismo, valor intensificador) contenida en algunas de las UF de la lengua origen (inglés) y por otro, los calcos o traducciones literales de otras UF.

Palabras clave: Fraseotraducción; enfoque multilingüe; competencia fraseológica; análisis traductológico contrastivo; corpus GRAFE; información pragmática.

Ramón MARTi SOLANO Un enfoque multilingüe y contrastivo de la traducción fraseológica: el corpus paralelo GRAFE 


\begin{abstract}
This paper presents the results of the translational analysis of a selection of phraseological units (PUs) in a sample taken from David Lodge's novels and from their translations into Spanish and French. A typology of the main problems of phraseotranslation is proposed by using a methodology based on the contrastive translational analysis by means of the GRAFE corpus and the concordancer Paraconc. They consist of linguistic features that are present in one of the target languages and that may absent in the other one or even in both. Two main phenomena are to be considered: on the one hand, the neutralization of the pragmatic information (language register, euphemisms, intensifying value) included in some PUs in the source language (English) and on the other hand, the calques or literal translations of other PUs.
\end{abstract}

Keywords: Phraseotranslation; multilingual approach; phraseological competence; contrastive translational analysis; GRAFE corpus; pragmatic information.

\title{
1. INTRODUCCIÓN
}

Las expresiones idiomáticas son, por regla general, difíciles de traducir pues implican dos niveles de interpretación, uno literal y otro figurado y, aún más importante, una conciencia lingüística de que se está frente a una unidad fraseológica (UF) no composicional para poder interpretarla correctamente. Además de este componente lingüístico-cognitivo, se apuntan otros dos, el componente cultural y el componente tecnológico (Mejri, 2008, pp. 247-249).

Si excluimos un gran número de UF que tienen una correspondencia exacta o casi exacta en la lengua meta, como take the bull by the horns > prendre le taureau par les cornes > "coger el toro por los cuernos" o read between the lines > lire entre les lignes $>$ «leer entre líneas» (Piirainen, 2012, 2016), muchas otras representan un verdadero desafío para el traductor. Bajo una apariencia normal se esconden expresiones en la lengua origen que pasan desapercibidas y que terminan por ser traducidas literalmente creando así enunciados que se prestan a interpretaciones variopintas muy lejanas de la significación fraseológica original. La competencia fraseológica constituye uno de los aspectos más desdeñados en el aprendizaje de una lengua extranjera; de ahí que se aconseje que los traductores dispongan de una buena competencia fraseológica con el fin de evitar las traducciones literales de las UF, práctica que suele ser bastante común (Xatara, 2002, 443).

Si trasladamos esta cuestión al campo de la traducción en general, y de la traducción literaria en particular, el nivel de dificultad aumenta por el simple hecho de que el traductor tiene que dominar y poner en relación los sistemas fraseológicos de la lengua origen (lengua extranjera) y de la lengua meta (lengua materna), lo que representa un ejercicio mental de una extrema complejidad, sobre todo teniendo en cuenta que tanto la imagen subyacente como los constituyentes de las UF de la lengua origen ejercen una influencia notable en la capacidad de decisión de los traductores.

Ramón MARTi SOLANO

Un enfoque multilingüe y contrastivo de la traducción fraseológica: el corpus paralelo GRAFE
CLINA

vol. 6-2, December 2020, 51-69

eISSN: 2444-1961

Ediciones Universidad de Salamanca - CC BY-NC-ND 


\section{LA FRASEOLOGÍA EN LA OBRA DE DAVID LODGE}

David Lodge es un autor británico cuyas novelas gozan de gran popularidad tanto en el Reino Unido como internacionalmente. Este universitario inglés ha sido profesor de literatura en la Universidad de Birmingham y sus novelas son un buen reflejo del mundo universitario en general, y del de los países anglosajones en particular. Hemos seleccionado las dos novelas que se encuentran en nuestro corpus paralelo GRAFE, Nice Work y Small World, que junto con Changing Places, forman una trilogía conocida como The Campus Trilogy. Este tipo de novelas, conocidas en inglés con la denominación de campus novels, ponen en relación a los personajes de los círculos universitarios entre ellos y con el mundo no universitario y sacan a la luz sus gozos y sus miserias con buenas dosis de humor y de ironía.

Las novelas de David Lodge son, desde el punto de vista lingüístico, el reflejo fiel de la lengua utilizada por la middle class británica (clase social acomodada con estudios universitarios compuesta principalmente por médicos, abogados y profesores que tienen buenos empleos e ingresos correspondientes). El propio David Lodge, prototipo de la middle class, utiliza en las partes narrativas y en las dialogadas un número importante de expresiones idiomáticas, lo que confiere a la narración y a los diálogos un aire de autenticidad y de naturalidad, características que se ven reforzadas por el uso de expresiones idiosincrásicas del inglés británico como, por ejemplo, move the goalposts ("cambiar las reglas del juego»), get on somebody's tits («hincharle las pelotas a alguien»), page three girl (culturema que designa la fotografía de una joven que suele aparecer con los pechos desnudos en la tercera página de los tabloides británicos), nail your colours to the mast ("tomar partido", "definirse»), the man on the Clapham omnibus («el hombre de la calle», «el ciudadano de a pie»), be thin on the ground ("escasear») y be sound as a bell («en perfectas condiciones»), entre muchas otras".

\section{TRADUCTOLOGÍA Y LINGÜÍSTICA DE CORPUS}

Además de los recursos de que disponen los traductores (diccionarios bilingües, traductores automáticos y memorias de traducción), habría que añadir los corpus paralelos y comparables, que pueden ser a su vez bilingües o multilingües. Por corpus paralelo se entiende un corpus de textos escritos en una lengua origen y su correspondiente traducción en la lengua meta (bilingüe) o las lenguas meta (multilingüe). Estos corpus paralelos o de traducción pueden ser muy útiles para comprobar tanto una intuición del traductor como el resultado de una búsqueda en el diccionario bilingüe, traductor automático o memoria de traducción.

1. Todas las traducciones al español de las unidades fraseológicas inglesas están tomadas del diccionario bilingüe Oxford Spanish Dictionary.

Ramón MARTi SOLANO

Un enfoque multilingüe y contrastivo de la traducción fraseológica: el corpus paralelo GRAFE
CLINA

vol. 6-2, December 2020, 51-69

elSSN: 2444-1961

Ediciones Universidad de Salamanca - CC BY-NC-ND 
Los corpus paralelos deben usarse con precaución pues, a veces, el análisis de los resultados puede inducir a error ya que estos últimos pueden estar influidos por la expresión en la lengua origen o por los mecanismos generales de traducción. Los corpus TenTen son un conjunto de corpus compuestos de textos extraídos de internet en más de cuarenta idiomas y que pueden consultarse en la plataforma digital Sketch Engine. Los corpus utilizados en este estudio para cotejar y comprobar las UF seleccionadas son el corpus inglés (enTenTen15), que cuenta con 15.703.895.409 palabras, el corpus español (esTenTen18), con 9.497.213.009, y el corpus francés (frTenTen12), con 9.889.689.889. Hemos utilizado igualmente, y con el mimo objetivo, los corpus paralelos EUROPARL7 (inglés, español y francés), y los corpus monolingües CORDE, CREA y CORPES, para el español, y el Corpus of Contemporary American English (COCA), para el inglés.

La propuesta metodológica que presentamos a continuación consiste en la utilización de una segunda traducción en otra lengua (preferentemente de la misma familia) para cotejar las divergencias y poder de esta manera identificar posibles errores de traducción. Este método no es infalible ya que el mismo error puede haberse producido en las dos lenguas meta pero, al menos, permite una primera revisión en la que ciertos aspectos lingüísticos pueden estar presentes en una lengua y ausentes en la otra.

\section{METODOLOGÍA DEL ESTUDIO: EL CORPUS GRAFE}

El corpus paralelo multilingüe GRAFE² permite la identificación, comparación y posterior análisis de traducciones en cinco idiomas europeos, a saber, griego, rumano, inglés, francés y español. Este corpus, producto de la colaboración entre las universidades de Poitiers y de Limoges, está compuesto de dos subcorpus, un subcorpus literario y otro subcorpus especializado (obras de lingüística general). El primero reúne una selección de novelas publicadas a partir de 1980 en las cinco lenguas del corpus y sus correspondientes traducciones en las otras cuatro lenguas. Hay que señalar que, por razones de derechos de autor, solo se han seleccionado pasajes del texto original y de sus traducciones de unas 17.000 palabras.

A partir de una selección manual de unidades fraseológicas en los textos ingleses, y gracias al programa de concordancias multilingüe ParaConc, se pudieron recabar las traducciones en francés y en español al estar todos los textos alineados.

La tabla 1 muestra la selección de segmentos de traducción seleccionados manualmente en las dos novelas. La gran mayoría de estos segmentos contienen expresiones idiomáticas, modismos o idiotismos que suelen ser locuciones verbales o

2. Más información sobre el corpus GRAFE (acrónimo de los nombres en francés de los cinco idiomas: grec, roumain, anglais, français, espagnol) en la página web <http://forellis.labo. univ-poitiers.fr/corpus-grafe-corpus-multilingue-grec-roumain-anglais-francais-espagnol/> (en francés).

Ramón Marti Solano

Un enfoque multilingüe y contrastivo de la traducción fraseológica: el corpus paralelo GRAFE
CLINA

vol. 6-2, December 2020, 51-69

elSSN: 2444-1961

Ediciones Universidad de Salamanca - CC BY-NC-ND 
nominales (to bear the brunt, the last straw). Existen, aunque en menor medida, colocaciones (to land a job, to keep house), binomios fraseológicos (on and off, the ebb and flow), comparaciones estereotipadas (as sound as a bell), proverbios (It's no use crying over spilt milk) y fórmulas rutinarias o rutinas conversacionales (what the blazes...?).

\begin{tabular}{|c|c|}
\hline Nice Work & Small World \\
\hline $\begin{array}{l}\text { a bone of contention } \\
\text { a step up the social ladder } \\
\text { all hell broke loose } \\
\text { as if by magic } \\
\text { bearing the brunt } \\
\text { by the same token } \\
\text { dying a natural death } \\
\text { gets on his tits } \\
\text { growing a beer belly } \\
\text { is landing a job } \\
\text { keeping a watchful eye on } \\
\text { let sleeping dogs lie } \\
\text { moves his bowels } \\
\text { moving the goalposts } \\
\text { needless to say } \\
\text { not by a long chalk } \\
\text { not know him from Adam } \\
\text { on and off } \\
\text { on song } \\
\text { to rub shoulders with } \\
\text { she had set her heart on } \\
\text { sick and tired } \\
\text { strictly speaking } \\
\text { the country is going to the dogs } \\
\text { the dust settled } \\
\text { the ebb and flow } \\
\text { the man on the Clapham omnibus } \\
\text { the top page three girl } \\
\text { the writing on the wall } \\
\text { then you'll be in trouble } \\
\text { they nailed their colours to the mast } \\
\text { to concede the point } \\
\text { to keep house } \\
\text { to put in an appearance } \\
\text { under the umbrella of } \\
\text { with mixed feelings } \\
\text { without putting on any airs and graces } \\
\text { you spoil that girl something rotten }\end{array}$ & $\begin{array}{l}\text { a bit thin on the ground } \\
\text { a free hand } \\
\text { as sound as a bell } \\
\text { coming to a head } \\
\text { have second thoughts } \\
\text { having her leg pulled } \\
\text { I strained every nerve } \\
\text { It's no use crying over spilt milk } \\
\text { keeping a low profile } \\
\text { rumour has it that } \\
\text { the last straw } \\
\text { to answer a call of nature } \\
\text { to his consternation } \\
\text { to hit the nail on the head } \\
\text { to put Rummidge on the map } \\
\text { to shake the dust of Rummidge off my feet } \\
\text { under your nose } \\
\text { what the blazes }\end{array}$ \\
\hline
\end{tabular}

Tabla 1. Segmentos idiomáticos en los pasajes del corpus GRAFE de las novelas Nice Work y Small World de David Lodge

Ramón MARTI SOLANO Un enfoque multilingüe y contrastivo de la traducción fraseológica: el corpus paralelo GRAFE 


\section{PROBLEMAS DE FRASEOTRADUCCIÓN}

La fraseotraducción y la fraseotraductología son dos disciplinas conexas y complementarias que se concentran exclusivamente en los aspectos teóricos y aplicados relativos a la traducción de las UF. Por UF entendemos toda combinación de unidades léxicas que presenta las características de fijación morfosintáctica y no composicionalidad semántica. Se dividen en dos grupos principales: los sintagmas fraseológicos (colocaciones y locuciones) y los enunciados fraseológicos (pragmatemas y paremias) ${ }^{3}$.

Uno de los problemas que se han señalado en relación con la traducción fraseológica ha sido la "falta de naturalidad", es decir, el uso de expresiones en la lengua meta que resultan arcaicas o demasiado remilgadas «cuyo origen quizá esté en los diccionarios bilingües» (Newmark, 1995, pp. 46-47). Esta apreciación de Newmark, que da como ejemplo la traducción del segmento she relieved nature por «ella exoneró el vientre», se contradice con los resultados obtenidos en los análisis de expresiones traducidas en la obra de David Lodge tal como se verá más adelante.

En cuanto a la traducción lexicográfica, hemos utilizado los diccionarios bilingües Hachette \& Oxford $(H \& O)$ para el francés y el Oxford Spanish Dictionary (OSD) para el español.

Hemos identificado seis tipos de problemas relacionados con la traducción de las expresiones idiomáticas inglesas: la traducción de la intensidad, la traducción analítica de los binomios fraseológicos, la inequivalencia en el registro lingüístico, la incidencia en la traducción de la imagen o de uno de los constituyentes, las traducciones literales que no tienen en cuenta el sentido traslaticio y la traducción de los eufemismos.

\subsection{La traducción de la intensidad}

Uno de los aspectos más complicados en fraseotraducción es el reconocimiento del valor intensificador de algunas UP y el empleo de otra UP en la lengua meta que contenga el mismo valor. Se trata de un componente pragmático (la intensidad), y no de un componente semántico (el significado equivalente), que tiene que ser interpretado y traducido correctamente. El valor intensificador es raramente neutro y suele ir asociado con un registro de lengua coloquial, familiar y, en muchos casos, vulgar.

El ejemplo (1) muestra un uso particular (inserción de un elemento externo a la forma canónica, something) de la expresión inglesa spoil somebody rotten. El análisis traductológico contrastivo entre la traducción española (1a) y la francesa (1b) permite apreciar el mecanismo de traducción en francés, una construcción (en el sentido de la gramática de construcciones) que combina dos frases simples yuxtapuestas (la

3. Esta tipología es la utilizada por el grupo de investigación PhraseoNet de la Universidad de Santiago de Compostela. Recuperado el 12 de julio de 2020 de http://www. phraseonet.com/ es/unidades-fraseologicas.

Ramón MARTi SOLANO

Un enfoque multilingüe y contrastivo de la traducción fraseológica: el corpus paralelo GRAFE
CLINA

vol. 6-2, December 2020, 51-69

elSSN: 2444-1961

Ediciones Universidad de Salamanca - CC BY-NC-ND 
primera negativa y la segunda afirmativa) seguidas del argumento en posición final. Contribuyen al valor intensificador no solo la construcción sintáctica y el argumento desplazado y focalizado sino los dos constituyentes léxicos principales y su relación sintáctica: el verbo gâter («mimar») y el verbo pourrir («mimar en exceso»).

(1) "Why can't she go on her own? A girl of seventeen should be able to go to the doctor's without someone to hold her hand.»

"I don't go in with her, not unless she wants me to. I just wait with her.»

Vic regards his wife suspiciously. "You're not going shopping with her afterwards?» Marjorie blushes. "Well, she needs a new pair of shoes...»

"You're a fool, Marje!» Vic exclaims. "You spoil that girl something rotten. All she thinks about is clothes, shoes, hairstyles.»

(1a) - ¿Y por qué no puede ir ella sola? Una chica de diecisiete años debería poder ir a ver al médico sin que nadie la llevara de la mano.

- Es que yo no entro con ella, a menos que así lo quiera. Me limito a esperarla.

Vic miró a su esposa con suspicacia.

- ¿Y no irás de compras con ella, después?

Marjorie se sonroja.

-Bueno, necesita unos zapatos nuevos...

- ¡Eres tonta, Marje! - exclama Vic-. Estropearás a esa chica con tanto mimo. Sólo piensa en vestidos, zapatos y peinados.

(1b) - Une fille de dix-sept ans est quand même bien capable d'aller chez le médecin sans qu'on la prenne par la main.

- Je ne rentre pas dans le cabinet avec elle, sauf si elle me le demande. Je reste seulement avec elle dans la salle d'attente.

Vic regarde sa femme d'un air soupçonneux. Tu ne vas pas faire des courses avec elle après?

Marjorie rougit. Il faut bien, elle a besoin d'une nouvelle paire de chaussures...

- Tu es une imbécile, Marje !, s'exclame Vic. Tu ne la gâtes pas, tu la pourris, ta

fille. Elle ne pense qu'aux fringues, aux chaussures et à ses cheveux.

La traducción española carece del doble valor intensificador de la expresión original (por un lado, el intensificador lexicalizado rotten y, por otro lado, el intensificador de registro coloquial something) y de la traducción francesa. A diferencia del español, el verbo francés pourrir comparte con el adjetivo inglés rotten el mismo sentido figurado aplicado a personas para indicar que son insoportablemente malcriadas. El verbo «estropear» no suele aplicarse en español a personas y, en cualquier caso, no significa «mimar», «Consentir» O «malcriar».

El H\&O da come traducción pourrir qn («consentir») y el OSD «mimar demasiado a alguien», «malcriar a alguien». Tanto en el primer caso como en el segundo el valor intensificador está presente en las traducciones lexicográficas.

Ramón MARTi SOLANO

Un enfoque multilingüe y contrastivo de la traducción fraseológica: el corpus paralelo GRAFE
CLINA

vol. 6-2, December 2020, 51-69

elSSN: 2444-1961

Ediciones Universidad de Salamanca - CC BY-NC-ND 
En el mismo sentido, se aprecia un valor intensificador superior en el caso de la traducción francesa (2b) con respecto a la traducción española (2a) de la expresión inglesa strain every nerve, tal como aparece utilizada en (2).

(2) «I'm sure l've heard that name before."

"l should hope so. What's yours?»

«Persse McGarrigle, from Limerick. Aren't you giving a paper this afternoon?» he said. «Title to be announced?»

«Right, Percy. That's why I strained every nerve to get here. Look at the bottom of the list. There are never many zees."

(2a) - Estoy seguro de haber oído antes este nombre.

-Quiero esperar que sí. ¿Cuál es el suyo?

-Persse McGarrigle, de Limerick. ¿No va usted a dar una conferencia esta tarde?

-inquirió-. ¿Título todavía por anunciar?

-Eso es, Percy. Por ello he apretado de firme para llegar aquí. Mire al final de la lista. Nunca suele haber muchas zetas.

(2b)- Je suis sûr d'avoir entendu ce nom quelque part.

- J'espère bien que oui. Quel est le vôtre?

- Persse McGarrigle, de Limerick. Vous ne devez pas donner une communication cet après-midi ? dit-il. Le titre sera annoncé ultérieurement?

- Exact, Percy. C'est pour ça que je me suis mis en quatre pour être ici. Regardez au bas de la liste. II n'y a jamais tant de z que ça.

El OSD da como traducción para la expresión strain every nerve «hacer un gran esfuerzo o un esfuerzo sobrehumano». Por su parte, el H\&O traduce la expresión con un verbo simple: s'évertuer (afanarse). El Oxford English Dictionary (OED) define la expresión como to make a great physical effort; to make the utmost effort («hacer un gran esfuerzo físico»; «hacer el máximo esfuerzo»). Una traducción fraseológica funcionalmente equivalente podría haber sido «hacer lo imposible».

\subsection{Traducción analítica de binomios fraseológicos}

El valor intensificador de muchos binomios fraseológicos en inglés se encuentra en la asonancia, aliteración y rima entre sus dos constituyentes, como ocurre en hustle and bustle, groan and moan o huff and puff. En otras ocasiones, la intensidad no se vale de mecanismos fonomorfémicos sino que utiliza combinaciones en las que el constituyente no composicional refuerza el valor del otro constituyente a lectura composicional. Este es el caso del binomio sick and tired (3), en el que el primer constituyente, sick, añade un fuerte valor de intensidad al segundo, tired, que significa aquí "cansado", en el sentido de «harto". El OED define el adjetivo sick en su quinta acepción como thoroughly tired or weary of a thing («extremadamente cansado o harto de

Ramón MARTi SOLANO

Un enfoque multilingüe y contrastivo de la traducción fraseológica: el corpus paralelo GRAFE
CLINA

vol. 6-2, December 2020, 51-69

elSSN: 2444-1961

Ediciones Universidad de Salamanca - CC BY-NC-ND 
alguna cosa») y añade que este mismo sentido aparece en las expresiones sinónimas sick and tired of o sick to death of. Se trata pues de una repetición que refuerza la intensidad que está ya presente en el propio adjetivo sick.

(3) Well, she was sick and tired of the place, tired of its beautiful architecture housing vanity and paranoia, glad to exchange its hothouse atmosphere for the real if smoky air of Rummidge.

(3a) Pues bien, ella estaba cansada, harta del lugar, cansada de su hermosa arquitectura que alojaba la vanidad y la paranoia, contenta de cambiar su atmósfera de invernadero por el aire real, aunque cargado de humo, de Rummidge.

(3b)Eh bien, elle en avait vraiment assez de cet endroit, assez de cette architecture superbe qui servait de refuge à la futilité et à la paranoïa, et elle était ravie d'échanger cette atmosphère de serre chaude contre l'air plus authentique, bien que plus enfumé, de Rummidge.

El OSD da como traducciones «estar absolutamente harto» o «estar hasta la coronilla» (fam.). Una búsqueda en el Corpus of Contemporary American English (COCA) de sick and [j] da como primer resultado sick and tired con 1.791 casos, seguido de sick and dying con solo 130 y, en tercer lugar, de sick and twisted, con 59 casos, lo que demuestra, desde el punto de vista cuantitativo, el carácter fijo y colocacional de la combinación, su unidad sintáctica y semántica. La traducción española (3a), influida por el carácter binomial de la expresión inglesa, repite dos adjetivos sinónimos, "cansado» y «harto», sin tener en cuenta la información pragmática complementaria que recogen tanto el OED como el OSD. La traducción francesa (3b) no incurre en este error y utiliza la expresión en avoir (vraiment) assez ("estar absolutamente harto»).

El corpus paralelo EUROPARL7 (inglés) proporciona 16 casos de sick and tired. La traducción mayoritaria en español es el adjetivo «harto» y en francés la UP en avoir assez («estar harto»). En ambos casos la traducción mayoritaria no se ve influida por el carácter binomial de la UF inglesa.

Además del valor intensificador que tienen muchos binomios fraseológicos, otros combinan dos constituyentes que pueden pertenecer o no al mismo campo semántico para indicar una sola y única realidad, concepto, idea o sentimiento.

(4) In his day a College of Advanced Technology, it has since grown in size and been raised to the status of a university, but without putting on any airs and graces.

(4a) En sus tiempos había sido Instituto de Tecnología Avanzada, y desde entonces había aumentado en tamaño y había adquirido la categoría de universidad, pero sin que ello le añadiera ningún carácter y ninguna gracia.

(4b)De son temps, ce n'était qu'un Institut supérieur de technologie, mais l'établissement s'était développé depuis et s'était élevé au rang d'université, sans, pour autant, prendre des airs distingués ou hautains.

Ramón MARTi SOLANO Un enfoque multilingüe y contrastivo de la traducción fraseológica: el corpus paralelo GRAFE
CLINA

vol. 6-2, December 2020, 51-69 elSSN: 2444-1961

Ediciones Universidad de Salamanca - CC BY-NC-ND 
El binomio airs and graces se utiliza en inglés como argumento de los verbos put on ("adoptar») o give oneself ("darse») y significa, en estos casos concretos, "darse aires». El OSD traduce airs and graces como «afectación» y su sentido peyorativo aparece de manera explícita en el OED en el marcador pragmático depreciative. La expresión suele usarse con un referente humano, aunque en (4) el referente es un centro de enseñanza superior. Lo mismo ocurre en español con la expresión «darse aires", aplicada comúnmente a personas. De nuevo, y como en el ejemplo anterior de sick and tired (3), la traducción española se ve influida por el carácter binomial de la UF de la lengua origen e incurre no solo en el calco semántico ${ }^{4}$ sino también en el calco sintáctico (4a) mientras que la traducción francesa opta por el reforzamiento del valor despreciativo por medio de la utilización de los sinónimos, distingué («distinguido») y hautain ("altivo", "altanero»).

\subsection{Inequivalencia en el registro lingüístico}

La inequivalencia en el registro lingüístico se produce en el componente connotativo-pragmático debido a la aparición de un lexema de distinto registro en una lengua y no en la otra. (Mellado Blanco, 2015, p. 167). En la UF get on somebody's tits (5) el único constituyente nominal es una palabra malsonante considerada vulgar en inglés, a saber, tits («tetas»). Habría que esperar, por consiguiente, que esta expresión inglesa correspondiese a otra expresión igualmente considerada vulgar en la lengua meta. Así, el OSD da como traducción «hincharle las pelotas a alguien» y la marca como británica y vulgar, al igual que lo hace el OED.

(5) Vic is all in favour of backing Britain, but there are times when the Mail's windy chauvinism gets on his tits.

(5a) Vic se muestra siempre favorable a respaldar a Gran Bretaña, pero hay veces en que el descarado chauvinismo del Mail le saca de quicio.

(5b) Vic ne demande pas mieux que de miser sur la Grande - Bretagne, mais il y a des fois où le chauvinisme ampoulé du Daily Mail lui tape sur le système.

En el Diccionario fraseológico documentado del español actual (DFDEA) la locución "sacar de quicio [a alguien]" no presenta ninguna marca, ya sea con respecto al nivel de uso (coloquial, popular, vulgar, jergal, juvenil o literario), a la actitud del hablante (humorístico, despectivo, irónico), al ámbito de uso (administrativo), a la frecuencia

4. Conviene señalar que el morfema de plural en algunos sustantivos en inglés puede dar lugar a un cambio semántico con respecto al significado del mismo sustantivo en singular. Así ocurre con air y grace, como ocurre igualmente en español con «aire» y «aires». En el caso concreto de grace, el plural graces nada tiene que ver con ninguno de los sentidos de "gracia" en español sino más bien con «buenos modales».

Ramón MARTi SOLANO

Un enfoque multilingüe y contrastivo de la traducción fraseológica: el corpus paralelo GRAFE
CLINA

vol. 6-2, December 2020, 51-69

elSSN: 2444-1961

Ediciones Universidad de Salamanca - CC BY-NC-ND 
de uso (frecuente, hoy raro) o incluso a la extensión geográfica (regional). La locución española es, a todas luces, una locución neutra sin rasgos pragmáticos o diafásicos lo que no la convierte en un equivalente funcional de la locución origen: la UF get on sb's tits es una expresión típicamente británica considerada vulgar y ofensiva. Esta información diastrática no se encuentra en la traducción española «le saca de quicio» (5a), que conserva la intensidad, pero no la vulgaridad. La traducción francesa, lui tape sur le système, (5b) carece igualmente del componente diafásico fundamental para que la equivalencia sea funcionalmente completa.

\subsection{Incidencia en la traducción de la imagen o de uno de los constituyentes}

Uno de los escollos que tiene que superar el traductor lo constituye la imagen subyacente en la UF de la lengua origen. En el caso de los enunciados fraseológicos, y más concretamente en la fórmula oracional Let sleeping dogs lie (6), los constituyentes principales sleeping (participio de presente) y dogs (sustantivo) pueden influir negativamente en la traducción al tratarse de los dos elementos que concentran el contenido léxico de la lectura composicional de la UF.

(6) Discussion of Sandra's sex-life could easily stray into the area of his and Marjorie's sex-life, or rather the lack of it, and he would rather not go into that. Let sleeping dogs lie.

(6a) La discusión sobre la vida sexual de Sandra podría derivar fácilmente hacia la zona de la vida sexual de él y Marjorie, o, mejor dicho, la carencia de la misma, y él prefiere no entrar en ese tema. Es mejor dejar que ciertas cosas duerman.

(6b) Toute discussion sur la vie sexuelle de Sandra pourrait bien déboucher sur un autre sujet, celui de leur vie sexuelle à tous les deux, ou plutôt de l'absence de vie sexuelle entre eux, et il préfère ne pas s'aventurer sur ce terrain. Pas la peine de réveiller les chiens qui dorment.

No se han encontrado casos del segmento «las/ciertas cosas duerman» (6a) en ninguno de los tres corpus de la Real Academia de la Lengua Española (CORDE, CREA y CORPES) ni tampoco en el corpus esTenTen18, lo que sugiere una creación improvisada del traductor influida por la UP de la lengua origen. El OSD, por su parte, proporciona como equivalentes «mejor no remover el asunto» $\mathrm{y}$ «mejor es no menearlo». El inglés y el francés (6b) comparten la misma imagen y las expresiones correspondientes gozan de una larga tradición en ambas lenguas ${ }^{5}$. El español, que no comparte esta

5. El Oxford Dictionary of Idioms explica que In the early 14th century the French phrase n'eveillez pas lou chien qui dort advised 'do not wake the sleeping dog', while Chaucer remarks

Ramón MARTi SOLANO Un enfoque multilingüe y contrastivo de la traducción fraseológica: el corpus paralelo GRAFE
CLINA

vol. 6-2, December 2020, 51-69

eISSN: 2444-1961

Ediciones Universidad de Salamanca - CC BY-NC-ND 
expresión con las otras dos lenguas, suele utilizar la paráfrasis «dejar las cosas como están», que mantiene el nombre genérico «cosas» y evita el influjo del verbo to sleep.

El ejemplo (7) muestra un caso muy común y conocido de colocación en inglés (mixed feelings). El OSD da como traducción de mixed feelings «sentimientos encontrados». Se trata de una colocación en la que el colocativo (mixed) adquiere un sentido particular en combinación con la base (feelings). La interferencia en este caso no es de la imagen, sino que es léxica: el participio pasado en función de adjetivo incide en la traducción y da como resultado «mezcla de sentimientos» (7a), que no significa lo mismo que «sentimientos encontrados». El tratamiento de esta unidad de traducción debe ser de colocación lexicalizada con fijación léxica y semántica y no de combinación libre de palabras. La traducción francesa (7b) no solo no cae en el error del calco léxico, sino que utiliza la colocación francesa equivalente, sentiment mitigé.

(7) Various bodies in Manufacturing Industry are working themselves into one of their regular lathers about the supposed low social esteem bestowed upon engineers and engineering. Vic reads this article with mixed feelings.

(7a) Varias entidades de la Industria Manufacturera se están ensarzando [sic] en una de sus disputas regulares sobre la supuestamente baja estimación social profesada a los ingenieros y a la ingeniería. Vic lee este artículo con una mezcla de sentimientos diversos.

(7b)Plusieurs organisations de l'Industrie manufacturière se sont émues comme d'habitude de la mauvaise image dont jouissent les ingénieurs et toute la profession sur le plan social. Vic lit l'article avec un sentiment plutôt mitigé.

El corpus paralelo EUROPARL7 (inglés) proporciona 78 casos de mixed feelings. Sus traducciones tanto en español como en francés son mayoritariamente «sentimientos encontrados" y sentiments mitigés respectivamente, soluciones que coinciden con las traducciones en los dos diccionarios bilingües utilizados en este estudio.

\subsection{Traducciones literales que no tienen en cuenta el sentido traslaticio}

Durante el estudio realizado hemos podido observar diversos casos de traducciones literales de UP inglesas. La competencia fraseológica está considerada como una de las competencias más difíciles de adquirir cuando se emprende el estudio de

in Troilus and Criseyde 'it is nought good a slepyng hound to wake'. The present form of the proverb seems to be traceable to Walter Scott's novel Redgauntlet (1824). El ejemplo más antiguo encontrado en el corpus Frantext remonta a 1433 y se encuentra en la obra titulada Complaintes de Charles d'Orléans. Se trata del uso idiomático de la expresión sans reveiller le chat qui dort en donde el constituyente nominal es chat ("gato») y no chien ("perro»).

Ramón MARTi SOLANO

Un enfoque multilingüe y contrastivo de la traducción fraseológica: el corpus paralelo GRAFE
CLINA

vol. 6-2, December 2020, 51-69

elSSN: 2444-1961

Ediciones Universidad de Salamanca - CC BY-NC-ND 
una lengua extranjera: los ejemplos (8a) y (8b) son reveladores del desconocimiento fraseológico tanto del traductor español como del traductor francés que optan ambos por una traducción literal. Es importante distinguir entre los calcos fraseológicos consagrados por el uso y las traducciones literales de UF que deben considerarse como casos de interferencia entre la lengua origen y la lengua meta (Sanz-Villar 2018, p. 76). Con respecto a la equivalencia en traducción fraseológica, González Rey (2014, p.235) señala los diferentes procedimientos de traducción indirectos y distingue, igualmente, entre el calco y la traducción literal.

La UP move the goalposts, utilizada en (8), la traduce el OSD por "cambiar las reglas de juego" y el $H \& O$ por changer les règles du jeu, un ejemplo evidente de laguna fraseológica en estas dos lenguas romances y de especificidad del inglés. Por su parte, el OED define esta expresión de la manera siguiente: «to move (also shift) the goalposts and variants: to alter the terms of a procedure, agreement, etc., before its completion or conclusion, esp. unfairly or covertly; to change the rules or requirements for something", definición en la que se observa la inclusión de un elemento informativo semántico-pragmático fundamental, a saber, el carácter arbitrario, injusto e interesado de la acción.

El uso de corpus paralelos permite verificar las traducciones más frecuentes y descartar otras: 6 de los 11 casos de move the goalposts en la versión inglesa de EURO$P A R L 7$ se traducen en español por "cambiar las reglas del juego". Tanto en (8a) como en (8b), la expresión ha sido traducida como si se tratase de una frase producto de la sintaxis libre, cuando en realidad estamos ante un claro ejemplo de UF con sentido figurado: cambiar de sitio la portería debe interpretarse en este contexto como alterar las normas o las reglas del juego para su propio beneficio.

(8) You only have to drive through the West Midlands to see that if we are in the Super-League of top industrial nations, somebody must be moving the goalposts.

(8a) Basta con viajar en coche a través de los Midlands occidentales para ver que, si nos encontramos en la superliga de las grandes naciones industriales, alguien debe de estar moviendo las porterías del campo.

(8b)II suffit de traverser les Midlands de l'Ouest en voiture pour comprendre que si nous sommes dans le tableau d'honneur des nations les plus industrialisées, quelqu'un a dû déplacer les poteaux de buts.

Se han encontrado once casos de la UF move the goals en EUROPARL7 (inglés). La traducción mayoritaria en el corpus paralelo español es «cambiar las reglas del juego" y en el francés, changer les règles du jeu, lo que refleja, en este caso concreto, una equivalencia fraseológica interlingüística total y coincidente con las traducciones en los diccionarios.

Otro caso flagrante de traducción literal se origina a partir de la expresión the man on the Clapham omnibus (9), expresión típicamente inglesa, de origen londinense, pues Clapham es un barrio situado al suroeste del centro de la capital (Reeds, 2006, p.

Ramón MARTi SOLANO

Un enfoque multilingüe y contrastivo de la traducción fraseológica: el corpus paralelo GRAFE
CLINA

vol. 6-2, December 2020, 51-69

elSSN: 2444-1961

Ediciones Universidad de Salamanca - CC BY-NC-ND 
443). La imagen es la de un señor cualquiera, como los que se ven en el autobús que se dirige a un barrio cualquiera y que representa, como lo registra el OSD, "al hombre de la calle» o «al ciudadano de a pie», o como lo traduce el H\&O, Monsieur tout le monde. Queda patente el carácter idiomático de la UP inglesa que les pasa desapercibido a ambos traductores, pues se trata de una expresión que hace referencia a un aspecto toponímico propio de la lengua origen y que carece de correspondencia conceptual fija en la lengua meta (Mogorrón Huerta, 2008, p. 392).

(9) For a few weeks the controversy featured in the national and even international press, up-market newspapers carrying spicy stories about the leading protagonists and confused attempts to explain the difference between structuralism and poststructuralism to the man on the Clapham omnibus.

(9a) Durante unas semanas, la controversia apareció en la prensa nacional e incluso en la internacional, con periódicos de gran circulación publicando historias picantes sobre los protagonistas principales e intentando explicar confusamente la diferencia entre estructuralismo y postestructuralismo [sic] al hombre que leía el diario en el autobús de Clapham.

(9b)Pendant quelques semaines, la controverse trouva un écho dans la presse nationale et même internationale, des journaux sérieux racontèrent des histoires épicées sur les principaux protagonistes et tentèrent confusément d'expliquer la différence entre le structuralisme et le poststructuralisme au banlieusard qui prenait l'omnibus de Clapham.

Hemos utilizado el buscador en línea Google Books Ngram Viewer para las UF que no tienen ocurrencias en EUROPARL7 (inglés). Solo se ha encontrado un caso de traducción de la UF the man on the Clapham omnibus en la versión española de la novela Dunkirk (Dunkerque) de Joshua Levine donde aparece traducido como «un pasajero cualquiera del ómnibus de Clapham».

Un último ejemplo de traducción literal que no tiene en cuenta el sentido traslaticio de la UF de la lengua origen lo encontramos en nail your colours to the mast (10), expresión que posee un origen especializado, concretamente naval, en la que la forma plural colours adquiere un sentido particular al tratarse de una metonimia para indicar la insignia de un barco 6 . La metaforización de «clavar su insignia en el mástil» da lugar a un sentido figurado que, aplicado a personas, designa el hecho de declarar, hacer público o dejar claro (normalmente una actitud, un sentimiento, una inclinación, una forma de entender la vida).

6. El DLE describe la voz «insignia» en su sexta acepción como «Bandera de cierta especie que, puesta al tope de uno de los palos del buque, denota la graduación del jefe que lo manda o de otro que va en él».

Ramón MARTi SOLANO

Un enfoque multilingüe y contrastivo de la traducción fraseológica: el corpus paralelo GRAFE
CLINA

vol. 6-2, December 2020, 51-69

eISSN: 2444-1961

Ediciones Universidad de Salamanca - CC BY-NC-ND 
(10)By living in what their parents called sin, they nailed their colours to the mast of youth revolt, while enjoying the security and mutual support of old-fashioned matrimony.

(10a) Al vivir en lo que sus padres calificaban de «pecado", izaban su estandarte en el mástil de la rebelión juvenil, al tiempo que disfrutaban de la seguridad y del apoyo mutuo de un matrimonio a la antigua.

(10b) Vivant dans ce que leurs parents appelaient le péché, ils se rassemblaient sous l'étendard de la révolte des jeunes, ce qui ne les empêchait pas de profiter de la sécurité et de l'aide mutuelle que procure le bon vieux mariage.

El OSD da como traducciones «tomar partido» y «definirse», traducciones que no se ajustan al contexto del ejemplo (12) y el H\&O, afficher ses opinions (une fois pour toutes) («mostrar sus opiniones [de una vez por todas]»). No solo la UF nail your colours to the mast se ha traducido literalmente en (10a) sino que además no existe ningún caso de la secuencia «estandarte en el mástil» en ninguno de los tres corpus del español consultados, lo que corrobora la literalidad de la traducción y la falta de naturalidad de esta secuencia en español, cuyo sentido resulta extremadamente difícil de interpretar si no se conoce el significado de la expresión original inglesa. La traducción francesa, por su parte, hace uso de una expresión consagrada en francés, se rassembler sous l'étendard de («militar debajo de la bandera de»).

La búsqueda en Google Books Ngram Viewer proporciona dos ejemplos de traducción palabra por palabra de la UF nail one's colours to the mast, concretamente en las versiones españolas de Doves of War (Palomas de Guerra) de Paul Preston y en Redcoat (Casaca roja) de Bernard Cornwell, donde se traducen por "clavar sus colores al mástil». El corpus EUROPARL7 (inglés) proporciona veintiún casos de esta UF. Resulta interesante constatar que, a diferencia de lo que ocurre con otras UF, esta expresión no cuenta con ninguna traducción mayoritaria en español y por otro lado solo se contabilizan una traducción con «tomar partido» y una con «definirse», las dos soluciones propuestas por el diccionario bilingüe. En francés sí que se da una traducción mayoritaria: donner/faire connaître/proclamer sa position («dar/dar a conocer/proclamar su posición»), traducción que no coincide con la propuesta por el diccionario bilingüe francés.

\subsection{Traducción de los eufemismos}

Las UF cumplen diversas funciones pragmáticas y discursivas y «en ellas priman las connotaciones expresivas, apreciativas, humorísticas, irónicas, despectivas, corteses y eufemísticas» (Mira Álvarez, 2011, p. 111). El valor eufemístico de ciertas UF debe ser traducido en la lengua meta con una UF equivalente tanto en su sentido denotativo como en su sentido connotativo. La UF inglesa move your bowels (11) es

Ramón MARTi SOLANO Un enfoque multilingüe y contrastivo de la traducción fraseológica: el corpus paralelo GRAFE
CLINA

vol. 6-2, December 2020, 51-69 elSSN: 2444-1961

Ediciones Universidad de Salamanca - CC BY-NC-ND 
una expresión eufemística utilizada comúnmente, entre muchos otros contextos extralingüísticos, por el personal médico para indicar a los pacientes la acción designada por el verbo «excretar» O «defecar». Las unidades fraseológicas españolas equivalentes son «mover/descargar/exonerar el vientre» o simplemente «hacer de vientre».

(11)By tacit agreement, Vic customarily moves his bowels in here, while Marjorie uses the guest cloakroom off the front hall, so that the atmosphere of the en suite bathroom remains unpolluted.

(11a) Por un acuerdo tácito, Vic suele ejercitar allí sus intestinos, en tanto que Marjorie utiliza el aseo de huéspedes ante el vestíbulo frontal, a fin de que la atmósfera del cuarto de baño en suite se mantenga impoluta.

(11b) Mais, par un accord tacite entre eux, Vic déleste toujours ses intestins ici, alors que Marjorie utilise les toilettes des invités qui donnent sur le hall d'entrée ; ainsi, l'atmosphère de la salle de bains contiguë demeure-t-elle toujours pure.

Move your bowels es una colocación cuya base es bowels y cuyo colocativo es move. Como en la mayoría de las colocaciones del tipo verbo + argumento, el verbo adquiere un significado colocacional que da lugar a una lectura no composicional del conjunto. En (11a) una traducción directa del sustantivo bowels («intestinos») obliga al traductor a forzar la traducción de la colocación dando lugar a la combinación «ejercitar los intestinos» de la cual no se han encontrado casos en ninguno de los tres corpus del español de la Real Academia Española.

Las UP homónimas o ambiguas (García-Page Sánchez, 2008, pp. 389-392) constituyen un motivo constante de interferencia entre la lectura no composicional y composicional de las UF. La locución nominal inglesa the call of nature (12) es una expresión eufemística utilizada con intención humorística para indicar, como en el ejemplo (11), la acción de «excretar» 0 «defecar».

(12) For more elaborate ablutions, or to answer a call of nature, it was necessary to venture out into the draughty and labyrinthine corridors in search of one of the communal washrooms, where baths, showers and toilets were to be found - but little privacy, and unreliable supplies of hot water.

(12a) Para unas abluciones más completas, o para responder a una llamada de la naturaleza, era necesario aventurarse por los laberínticos y ventosos pasillos en busca de uno de los cuartos de baño comunitarios, en los que cabía encontrar bañeras, duchas e inodoros, pero poco aislamiento y un suministro escasamente fiable de agua caliente.

(12b) Si l'on désirait des ablutions plus élaborées, ou si l'on souhaitait satisfaire des besoins naturels, il fallait s'aventurer dans un dédale de couloirs pleins de courants d'air avant de dénicher l'une des salles de bains collectives où l'on pouvait trouver baignoires, douches et W.-C. mais malheureusement bien peu d'intimité et un débit d'eau chaude capricieux.

Ramón MARTI SOLANO

Un enfoque multilingüe y contrastivo de la traducción fraseológica: el corpus paralelo GRAFE
CLINA

vol. 6-2, December 2020, 51-69

elSSN: 2444-1961

Ediciones Universidad de Salamanca - CC BY-NC-ND 
El traductor español ha pasado de largo y no ha sabido captar el sentido de la expresión inglesa contrariamente al traductor francés que utiliza la correspondiente expresión francesa faire ses besoins («hacer sus necesidades»). A call of nature es una UF cuando tiene el sentido humorístico de «necesidad imperiosa de hacer sus necesidades». En español, esta combinación léxica no es solo insignificante en los corpus (ningún caso en el CORDE, solo 2 en el CREA y 5 en el CORPES) sino que además no tiene, ni por asomo, el sentido figurado y humorístico que posee la expresión inglesa.

Una búsqueda en Google Books Ngram Viewer permite comprobar que la UF a call of nature ha sido traducida literalmente en otras obras literarias. Hemos encontrado dos ejemplos en obras traducidas, concretamente en las versiones españolas de Blood of the Caesars (La maldición de los Césares) de Stephen Dando-Collins y en On Canaan's Side (En el lado de Canaán) de Sebastian Harry.

\section{CONCLUSIONES}

La selección de unidades fraseológicas en inglés y el análisis de sus traducciones en español y en francés han permitido revelar los principales problemas relacionados con la fraseotraducción. Estos son la neutralización del valor intensivo original, la traducción analítica de los binomios fraseológicos, la inequivalencia en el registro lingüístico, la incidencia en la traducción de la imagen o de uno de los constituyentes del fraseologismo original, las traducciones literales y la neutralización del valor eufemístico. Los resultados de este estudio ponen de manifiesto, sobre todo, el número importante de fraseologismos ingleses que se traducen literalmente al igual que preconiza la utilización de un corpus multilingüe para indagar en los fenómenos traductológicos gracias a la comparación entre las traducciones de dos lenguas meta. Es evidente que sería necesario un estudio de mayor extensión y calado para poder establecer una tipología más exhaustiva de los principales problemas de fraseotraducción. En cualquier caso, son dignos de señalar dos fenómenos interesantes: por un lado, la neutralización de los valores pragmáticos (intensidad, registro de lengua, eufemismo) presentes en las expresiones en inglés y, por otro lado, el número importante de expresiones que no son reconocidas como tales y que acaban siendo traducidas de forma literal. El corpus paralelo GRAFE permite cotejar las actualizaciones discursivas de las UF en textos literarios traducidos, lo que constituye un recurso excepcional para las investigaciones lingüísticas en general y para los traductores y traductólogos en particular.

\section{BIBLIOGRAFÍA}

BARry, Sebastian. 2013. En el lado de Canaán. Barcelona: Alba Editorial. Cornwell, Bernard. 2017. Casaca roja. Madrid: Pàmies.

Ramón MARTi SOLANO

Un enfoque multilingüe y contrastivo de la traducción fraseológica: el corpus paralelo GRAFE
CLINA

vol. 6-2, December 2020, 51-69 eISSN: 2444-1961

Ediciones Universidad de Salamanca - CC BY-NC-ND 
Corpus de Referencia del Español Actual (CREA). Recuperado el 20 de noviembre de 2020 de http://corpus.rae.es/cordenet.html.

Corpus del Español del Siglo XXI (CORPES). Recuperado el 20 de noviembre de 2020 de http:// web.frl.es/CORPES/view/inicioExterno.view;jsessionid=261A80E2BB843FEDC53686AED833D93B.

Corpus Diacrónico del Español (CORDE). Recuperado el 20 de noviembre de 2020 de http:// corpus.rae.es/cordenet.html.

Corpus of Contemporary American English (COCA). Recuperado el 20 de noviembre de 2020 de https://www.english-corpora.org/coca/.

DANDo-Coluins, Stephen. 2009. La maldición de los Césares. Barcelona: Robinbook.

Diccionario de la Lengua Española. Recuperado el 20 de noviembre de 2020 de https://dle.rae. es/.

Diccionario Oxford español-inglés/inglés-español. 1994. Oxford: Oxford University Press.

García-PAge SÁnchez, Mario. 2008. Introducción a la fraseología española. Barcelona: Anthropos.

GonzÁLEZ REY, María Isabel. 2014. «Le “ double ” principe d’idiomaticité dans la traduction littéraire». Revista de Filología 32: 227-244.

Le Grand Dictionnaire Hachette-Oxford français-anglais - anglais-français. 2007. Oxford: Oxford University Press.

LeVINE, Joshua. 2017. Dunkerque. Madrid: HarperCollins Español.

LodGe, David. 1984. Small World. Londres: Penguin.

LODGE, David. 1988. Nice Work. Londres: Penguin.

LodGE, David. 1990. Jeu de société. [orig. Nice Work]. Traducido por Maurice Coutourier e Yvonne Coutourier. París: Rivages.

LODGE, David. 1996. jBuen Trabajo! [orig. Nice Work]. Traducido por Esteban Rimbau. Barcelona: Anagrama.

LoDGE, David. 2006. El mundo es un pañuelo. [orig. Small World]. Traducido por Esteban Riambau, Barcelona: Anagrama.

LodGE, David. 2013. Un tout petit monde. [orig. Small World]. Traducido por Maurice Coutourier e Yvonne Couturier. París: Rivages.

MEJRI, Salah. 2008. «Figement et traduction : problématique générale». Meta 53(2) : 244-252.

Mellado Blanco, Carmen. 2015. «Parámetros específicos de equivalencia en las unidades fraseológicas (con ejemplos del español y el alemán)». Revista de Filología 33: 153-174.

Mira ÁlvareZ, Germán Darío. 2011. «La equivalencia en la traducción de las unidades fraseológicas. Un estudio empírico». Wkala, revista de lenguaje y cultura 16(27): 105-131.

Mogorrón Huerta, Pedro. 2008. «Compréhension et traduction des locutions verbales». Meta 53(2) : 378-406.

Newmark, Peter. 1995. Manual de traducción. [orig. A Textbook of Translation]. Traducido por Virgilio Moya. Madrid: Cátedra.

Oxford English Dictionary. Recuperado el 20 de noviembre de 2020 de https://www.oed.com/.

PIIRAINEN, Elisabeth. 2012. Widespread Idioms in Europe and Beyond: Toward a Lexicon of Common Figurative Units. Nueva York: Peter Lang.

PIIRAINEN, Elisabeth. 2016. Lexicon of Common Figurative Units. Widespread Idioms in Europe and Beyond. Volume II. Nueva York: Peter Lang.

Preston, Paul. 2011. Palomas de Guerra. Madrid: Debolsillo.

Ramón MARTI SOLANO

Un enfoque multilingüe y contrastivo de la traducción fraseológica: el corpus paralelo GRAFE
CLINA

vol. 6-2, December 2020, 51-69

elSSN: 2444-1961

Ediciones Universidad de Salamanca - CC BY-NC-ND 
ReEDS, Nigel. 2006. A Word in your Shell-like. 6,000 Curious and Everyday Phrases Explained. Londres: Collins.

SANZ-VILLAR, Zuriñe. 2018. «Interference and the Translation of Phraseological Units in a Parallel and Multilingual Corpus». Meta 63(1): 72-93.

Seco, Manuel, Olimpia Andrés y Gabino Ramos. 2004. Diccionario fraseológico documentado del español actual. Locuciones y modismos españoles. Madrid: Aguilar.

Sketch Engine. Recuperado el 20 de noviembre de 2020 de https://www.sketchengine.eu/.

The Oxford Dictionary of Idioms. 2004. Oxford: Oxford University Press.

Xatara, Claudia Maria. 2002. «La traduction phraséologique». Meta 47(3): 441-444. 